\title{
Rechazos de exportaciones por incumplimiento de medidas no arancelarias: el caso de los productos agrícolas latinoamericanos en la frontera de Estados Unidos*
}

\author{
Export refusals for non-compliance with \\ non-tariff measures: the case of Latin \\ American agricultural products at the \\ United States border
}

\author{
Sofía Boza** \\ Juan Rozas ${ }^{* * *}$ \\ Rodolfo Rivers ${ }^{* * * *}$
}

\begin{abstract}
RESUMEN
El sector agrícola es de gran importancia para la estrategia comercial latinoamericana, siendo Estados Unidos uno de sus principales destinos. Los requisitos relativos a calidad e inocuidad alimentarias de dicho mercado se han incrementado y se reflejan en un número creciente de medidas no arancelarias. El incumplimiento de estas puede llevar al rechazo del cargamento en la frontera, lo que implica pérdidas importantes. El objetivo del presente artículo es contribuir a ampliar el conocimiento acerca de los rechazos de exportaciones agrícolas por incumplimiento de normas relativas a calidad e inocuidad alimentarias. Para ello se
\end{abstract}

\footnotetext{
* La investigación en el presente documento se desarrolló en el marco del proyecto "Programa de Estímulo a la Excelencia Institucional 20I4", del Instituto de Estudios Internacionales de la Universidad de Chile, financiado por la Vicerrectoría de Investigación y Desarrollo de dicha Casa de Estudios.

** Departamento de Economía Agraria, Facultad de Ciencias Agronómicas e Instituto de Estudios Internacionales, Universidad de Chile.sofiaboza@u.uchile.cl. Recibido el 27 de Diciembre de 20I7. Aceptado el 20 de junio de 2018.

**** Dirección General de Relaciones Económicas Internacionales de Chile.jrozas25@gmail.com.

******Ministerio de Salud de Chile. rrivers@gmail.com.
} 
consideró específicamente el caso de las hortalizas y frutas frescas procedentes de América Latina en la frontera de Estados Unidos.

Palabras clave: Comercio agrícola - Calidad e inocuidad alimentarias - Rechazos de exportaciones - América Latina - Estados Unidos.

\section{ABSTRACT}

The agricultural sector is of great importance for Latin American trade, with United States as one of its main markets. Unites States food quality and safety requirements have increased and are reflected in a growing number of non-tariff measures. Failure on the compliance of those requirements can lead to the refusal of the shipment at the border, implying significant losses. The aim of this paper is to contribute to a further understanding about the agricultural export refusals for non-compliance with food quality and safety requirements. For this, we considered the case of Latin American fresh fruits and vegetables at the United States border.

Keywords: Agricultural trade - Food quality and safety - Export refusals - Latin America - United State. 


\section{INTRODUCCIÓN}

En años recientes, el comercio internacional se ha caracterizado por el incremento de la participación de las medidas no arancelarias (Hoekman y Nicita, 2008; Santana y Jackson, 20I2; Nicita y Gourdon, 2013). Entre ellas están las de tipo técnico, referidas a los atributos de los productos de importación y sus procesos anexos (UNCTAD, 20I3). La imposición de estas restricciones a los productos agrícolas busca garantizar la calidad e inocuidad de los alimentos. Dichas restricciones enfrentan fallos de mercado como la asimetría de información entre oferta y demanda, la generación de externalidades negativas o la falta de provisión de bienes públicos (Van Tongeren et al., 2009; Hobbs, 2010).

El cumplimiento de las medidas no arancelarias de tipo técnico supone, en algunos casos, mayores costos de producción. Diversas investigaciones han concluido que, como consecuencia, este tipo de medidas puede limitar el comercio agroalimentario (Hoekman y Nicita, 2008; Disdier y Fontagné, 20I0; Disdier y Marette, 20I0; Beghin y Melatos, 20I2; Penello, 20I4). En contraste, otros autores sugieren que las medidas no arancelarias de tipo técnico constituyen un potencial estímulo para las exportaciones de aquellos productores capaces de cumplirlas (Wilson y Bray, 20ıо; Crivelli y Gröschl, 20I2; Ferro et al., 20I5).

Los países miembros de la Organización Mundial de Comercio (омC) establecieron el marco normativo multilateral al cual deben atender los requisitos técnicos impuestos a las importaciones, para propiciar que sean lo menos lesivos posibles para el comercio internacional. Dicho marco normativo quedó reflejado en el Acuerdo sobre Aplicación de Medidas Sanitarias y Fitosanitarias (Acuerdo MSF) y en el Acuerdo sobre Obstáculos Técnicos al Comercio (Acuerdo otc), ambos resultados de la Ronda de Negociaciones de Uruguay.

El Acuerdo MSF tiene por objetivo asegurar que los países miembros de la omc puedan imponer las medidas necesarias para garantizar la inocuidad de los alimentos, así como la salud de las personas, animales y vegetales, sin que ello se utilice con fines de proteccionismo comercial. El Acuerdo отс se focaliza en garantizar la potestad de los países de adoptar las medidas necesarias para asegurar la calidad de las exportaciones, la protección del medioambiente, la prevención de prácticas que puedan inducir a error, la protección de la seguridad nacional y los aspectos señalados en el Acuerdo MSF. La transparencia es uno de los principios presentes en ambos acuerdos. Este establece que los Miembros han de notificar a la Secretaría de la omc su intención de hacer entrar en vigor o modificar sus medidas (MSF/OTC), buscando con ello mayor claridad y previsibilidad. Entre 1995 y 20I2, los países miembros de la OMC informaron a la 
Secretaría la intención de hacer entrar en vigor I4.864 MSF y 15.650 OTC; Estados Unidos fue el país con mayor número de notificaciones realizadas en ambos casos, con 3.489 y 97I respectivamente (Boza y Fernández, 2016).

$\mathrm{El}$ incumplimiento (o indicio de incumplimiento) de los requisitos técnicos por el exportador en un mercado determinado puede llevar al rechazo del cargamento infractor ${ }^{1}$. La consecuencia última es la pérdida económica, sobre todo si se ordena su destrucción. La reincidencia en la detección de alimentos contaminados daña la credibilidad de los países, lo que afecta sus exportaciones (Jouanjean, 20I2). Dicha situación resulta especialmente compleja para economías en las que el comercio exterior de alimentos tiene un peso importante, como América Latina. La región es en la actualidad el mayor exportador neto de productos agrícolas a nivel mundial, con expectativa de crecimiento continuado a futuro (OECD y FAO, 20I5). Uno de los principales destinos de estos productos es Estados Unidos. Más de un tercio del valor total de las importaciones alimentarias estadounidenses corresponde a mercancías procedentes de América Latina. Dentro de lo anterior, los productos agrícolas con una mayor

1 Entendiendo, en adelante, cargamento conforme a la definición realizada por el Departamento de Agricultura de Estados Unidos, como un lote único de un determinado producto alimenticio ofrecido por un transportista determinado, en un lugar y tiempo precisos (Buzby et al., 2008). participación son frutas y hortalizas. El $40 \%$ y el $20 \%$, respectivamente, de su consumo en dicho país son abastecidos mediante la importación. Estos porcentajes han crecido de manera sostenida en las últimas décadas. No obstante, el ingreso de productos al mercado estadounidense resulta, así como una oportunidad, un desafío en términos del cumplimiento de los requisitos técnicos exigidos.

Los rechazos a las exportaciones no solamente cuestionan a los agentes privados involucrados, sino que es de esperar reflejen asimismo el funcionamiento de los sistemas nacionales de control de alimentos. En general, estos incorporan las políticas y la legislación alimentaria; la gestión del control de los alimentos, los laboratorios de diagnóstico y análisis; la inspección de los alimentos; la imposición del cumplimiento de las normas y la certificación; la preparación y respuesta ante situaciones de emergencia; la vigilancia de las enfermedades transmitida por los alimentos, y la información pública, la educación y la comunicación. La calidad de dichos sistemas está en su capacidad de desempeñar las funciones pertinentes de manera eficaz, eficiente y sostenible, con el fin de ofrecer alimentos inocuos para el consumo interno y la exportación (FAO, 2007).

El objetivo del presente artículo es contribuir a ampliar el conocimiento acerca de los rechazos de exportaciones agrícolas por incumplimiento de medidas no arancelarias relativas a calidad e inocuidad alimentarias. Para ello, 
se consideran específicamente las hortalizas y frutas frescas procedentes de los principales países exportadores de América Latina en la frontera de uno de los mercados de mayor relevancia para la región: Estados Unidos. Los resultados a continuación se presentan en las siguientes secciones: i) revisión general de los sistemas de control de alimentos en América Latina, ii) funcionamiento del sistema de inspección en frontera de Estados Unidos, iii) evolución de los rechazos en frontera de Estados Unidos de importaciones hortofrutícolas procedentes de América Latina y iv) consideraciones finales.

\section{Revisión de los sistemas de control de alimentos en América Latina}

En América Latina, la producción de alimentos tiende a ser atomizada, con numerosos productores no organizados y mercados no estructurados de pequeña escala. El sector alimentario está evolucionando rápidamente, con escaso apoyo para la introducción de nuevas tecnologías por parte de empresas micro, pequeñas y medianas. La industria de los alimentos está fragmentada e insuficientemente financiada, sumado a que el poder adquisitivo de la mayoría de los consumidores es aún bajo. Los marcos reglamentarios para la inocuidad de los alimentos a menudo son incompletos o anticuados, los sistemas adolecen de falta de capacidad técnica, institucional y de control de gestión; no hay políticas integradas ni programas comunes y existe falta de coordinación (FAO, 20I2). Respecto de esto último, una de las razones de la desarticulación es que los organismos de sanidad animal y vegetal fueron creados como un apoyo a la producción y con un mandato central focalizado en el control de las enfermedades de los animales y las plantas. Por lo tanto, la responsabilidad respecto de la vigilancia de las condiciones de calidad e inocuidad de los alimentos para el consumidor está distribuida entre varios organismos, incluidos aquellos en el ámbito de la salud humana, otros de la agricultura, industria, comercio, pesca, municipalidades, etc. (FAO, 2OIO).

No obstante, el escenario no es el mismo en todos los países, sino que resulta heterogéneo y en definición.

Existen grandes diferencias de complejidad y cobertura entre los sistemas nacionales que regulan el control de alimentos. Estas diferencias de capacidades, sumadas a la globalización de la cadena alimentaria y la necesidad de cumplir con requisitos más allá de lo nacional, ha generado brechas significativas en las velocidades de adecuación de las normas de control de alimentos según los países estén más enfocados al mercado de exportación o al mercado interno.

Respecto de la comparación entre países de América Latina, se pueden referir los resultados del proyecto "Asistencia para el diseño y/o fortalecimiento de políticas de inocuidad de alimentos para los países de la región" desarrollado entre 2010 y $20 \mathrm{II}$ por FaO. En él se evaluaron los sistemas 
nacionales de control de alimentos regionales conforme a cinco áreas generales: gestión, legislación, inspección, laboratorios y comunicación. Los países estudiados fueron: en el Caribe, Cuba y República Dominicana; en Centroamérica, Costa Rica, El Salvador, Honduras, Nicaragua y Panamá, y en Sudamérica, Argentina, Chile, Ecuador, Paraguay, Perú y Uruguay.

En Boza et al. (20I4) se sintetizan los resultados globales del estudio. La capacidad de control de alimentos a nivel regional es estimada en el 54,2\%, siendo la condición ideal un valor del I00\%. Sudamérica es la subregión mejor evaluada en todas las áreas consideradas. Como muestra el Gráfico I, los países que presentan mayor capacidad de control de alimentos son Chile (75\%) y Argentina (70\%). Por el contrario, países como Ecuador, Cuba, Honduras y El Salvador están incluso por debajo del promedio regional, no alcanzando a llegar al 50\% de capacidad.

\section{GRÁFICO I}

Comparación de capacidades regionales de control de alimentos (\% de logro)

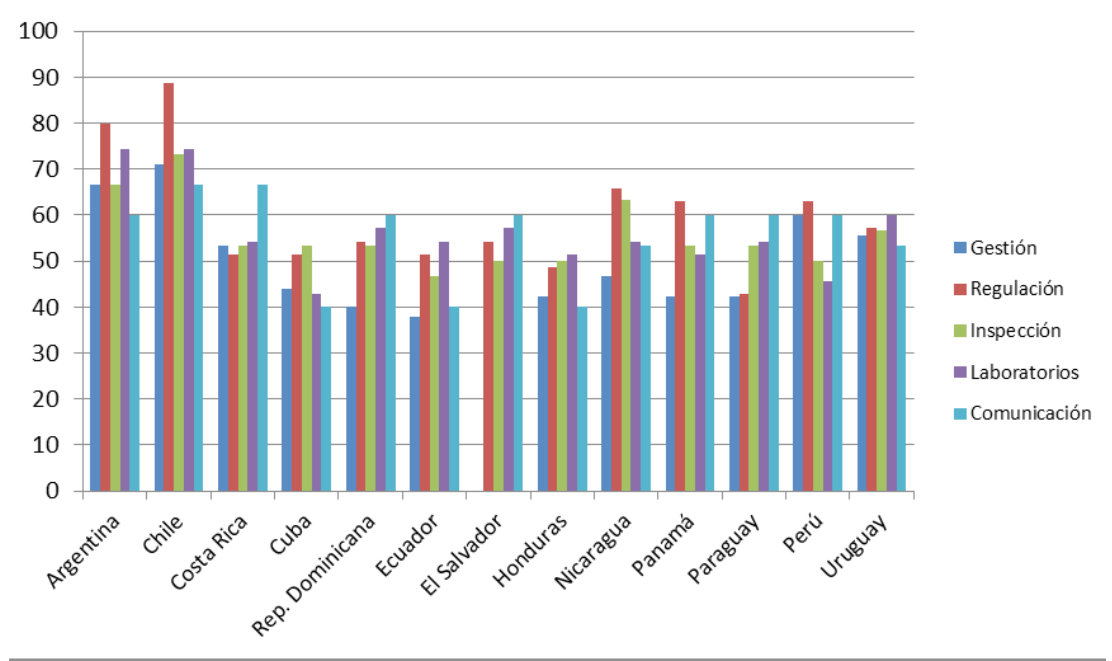

Fuente: Boza et al. (20I4) 
De forma complementaria, se agrupó a los países en el estudio de acuerdo a su nivel económico, tomando como referencia para ello la clasificación del Banco Mundial (ingreso alto, ingreso medio-alto, ingreso medio-bajo e ingreso bajo). Los resultados obtenidos muestran la existencia a priori de una relación directa entre el nivel de ingresos de un país y su capacidad de control de alimentos. Los países de ingreso alto tuvieron una capacidad de control de alimentos promedio de 65,67\%; los de ingreso medio-alto de $55,67 \%$, y los de ingreso medio-bajo del $49,48 \%$.

\section{GRÁFICO II}

Participación por país de América Latina en notificaciones MSF, 1995-2012

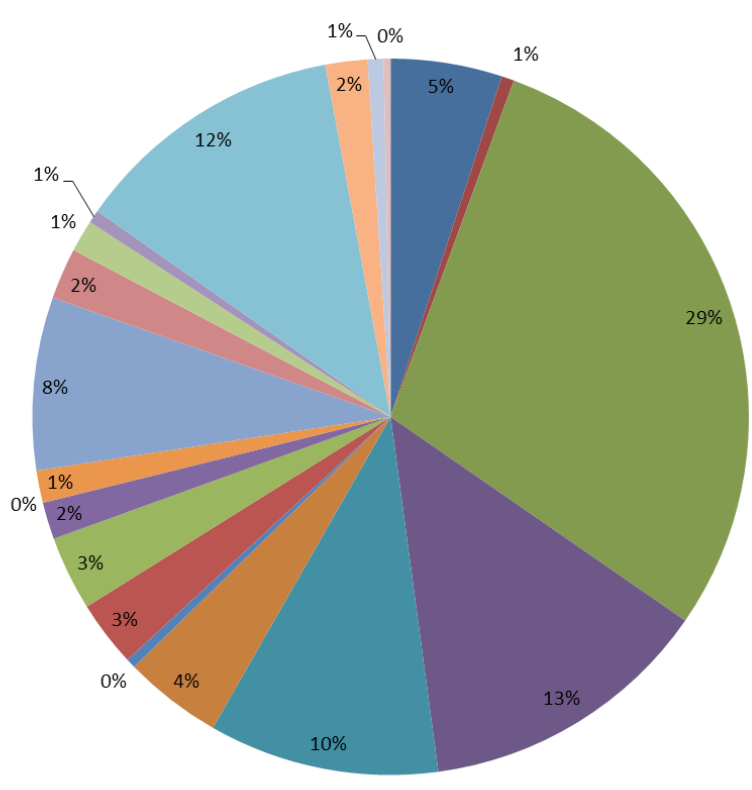

Fuente: Elaboración propia en base a WTO SPS Information Management System

Otra área relacionada en que se observan importantes diferencias entre países de América Latina es la actividad regulatoria, la cual se puede aproximar mediante el número de medidas sanitarias y fitosanitarias notificadas a la омc. Como muestra el Gráfico

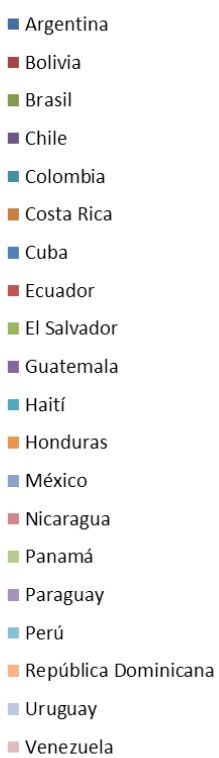

II, más del 70\% de las medidas notificadas por América Latina están concentradas en cinco países: Brasil, Chile, Colombia, México y Perú. Conforme a Boza y Muñoz (2017), estas diferencias están condicionadas precisamente por las capacidades técnicas y legales 
disponibles, y en menor medida por variables comerciales.

\section{Sistema de inspección en frontera de productos alimentarios en Estados Unidos}

La inspección de los productos alimentarios en frontera de Estados Unidos está esencialmente en manos de dos agencias federales: la Food and Drug Administration (FDA) y el Food Safety and Inspection Service (FSIS). La primera de ellas tiene jurisdicción sobre todo tipo de alimentos a ser ingresados en el país, excepto carnes (que no sean de caza ni exóticas), aves y procesados derivados del huevo. El control de estos productos le corresponde al Fsis. Dado el ámbito del presente artículo (productos agrícolas) nos centraremos, en adelante, en la FDA.

Hasta hace poco, el funcionamiento general de la FDA en materia de inocuidad de los alimentos se regía por la Ley Federal de Alimentos, Medicamentos y Cosméticos de 1938. Sin embargo, en 20II, el Presidente de los Estados Unidos firmó la nueva Ley de Modernización de la Inocuidad Alimentaria (FSMA). La entrada en vigor de la FSMA fue un reconocimiento de la necesidad de proporcionar a los organismos públicos medios adicionales para garantizar que los alimentos que se consumen no presenten riesgos para la salud. De hecho, estos riesgos se consideran un problema de salud pública, teniendo en cuenta la gran cantidad de casos de enfermedades transmitidas por los alimentos en Estados Unidos cada año. De acuerdo con Scallan et al. (20II) hay 9,4 millones de episodios de enfermedades transmitidas por los alimentos debido a los patógenos más comunes (por ejemplo, Norovirus, Salmonella spp.,Clostridium perfringens, Campylobacter spp., Ampylobacter spp. y Toxoplasma gondii). El énfasis de la FSMA está en las acciones preventivas. Por lo tanto, el enfoque ha pasado de mecanismos punitivos contra procedimientos incorrectos a incentivos para aquellos apropiados.

La FDA supervisa la inspección de la mayor parte de las importaciones de alimentos a Estados Unidos. No obstante, solo un I\% de los cargamentos es revisado por sus agentes debido a limitaciones operativas (Artecona y Flores, 2009); lo que lleva a dar prioridad a aquellos considerados más riesgosos. Los exportadores deben presentar un aviso de entrada de sus mercancías para facilitar el direccionamiento de las inspecciones y así aprovechar los recursos disponibles. Mediante el Operational and Administrative System for Import Support (oAsIs) se recogen y procesan las solicitudes de importación. La FDA toma la decisión de admitir el cargamento sin necesidad a priori de inspección, o establece que esta se realice en base a la información en OASIS (Grundke y Moser, 20I4).

Las inspecciones que realiza la FDA se dividen en dos tipos: exámenes de campo y de laboratorio. Los exámenes de campo se desarrollan en los puertos de entrada mediante pruebas 
organolépticas, correspondiendo a la mayor parte de las inspecciones. Dos posibles consecuencias se derivan de la detección de incumplimiento de las normas durante las inspecciones: la destrucción del cargamento, en la medida que su consumo sea peligroso, o la devolución del cargamento, el cual puede desviarse a otro mercado, lo que ha sucedido frecuentemente en casos como el de los productos del mar (Grant y Anders, 20II). Asimismo, el exportador tiene frente a la devolución del cargamento la posibilidad de reacondicionar la mercancía, para intentar nuevamente su ingreso a Estados Unidos (Artecona y Flores, 2009; Grundke y Moser, 20I4).

La FDA puede ordenar el rechazo de un cargamento incluso sin someterlo a inspección física en algunos casos específicos. Lo anterior sucede si se manejan antecedentes que hagan inferir que puede existir una violación de los requisitos, como la trayectoria de incumplimientos que tenga un país para un tipo de producto, lo que obliga al exportador a demostrar ante la FDA que su mercancía es segura (Becker, 20I0). El rechazo sin inspección permite una racionalización del número de revisiones a realizar, pero también puede derivar en mayor arbitrariedad.

\section{Rechazos de exportaciones}

latinoamericanas en la frontera de Estados Unidos

Para esta sección se utilizaron los registros en la base de datos OAsIs de la
FDA para el período de 2002 a 20I4. Los productos que se consideraron fueron hortalizas, frutas frescas y sus derivados, conforme a las categorías contenidas en los capítulos 07 y 08 del Sistema Armonizado de Designación y Codificación de Mercancías (SA). La focalización en productos hortofrutícolas se debe principalmente a dos motivos: el primero de ellos porque, dentro de los productos agrícolas, son los que han experimentado un mayor número de rechazos (Allen et al., 2008; Buzby et al., 2008; Artecona y Flores, 2009; Brooks et al., 2009; Buzby y Regmi, 2009). El segundo es porque concentran más de un tercio $(35,4 \%)$ del total de las exportaciones alimentarias ${ }^{2} \mathrm{de}$ América Latina hacia Estados Unidos, según datos en la base World Integrated Trade Solution (wits) del Banco Mundial de 2002 a 20I4. De hecho, la creciente presencia de América Latina como proveedor de hortofrutícolas para Estados Unidos, es un fenómeno que se viene observando ya desde los años noventa (Brooks et al., 2009).

Dada la gran cantidad de registros en la base oAsIs, se decidió priorizar el estudio de aquellos países de la región con mayor relación comercial con Estados Unidos en el ámbito de los productos hortofrutícolas. Para seleccionar dichos países se recurrió a la información en la ya mencionada base wits. México es, con amplia diferencia,

2 A efectos del presente artículo, dicha denominación abarca los capítulos I al 24 del Sistema Armonizado. 
el principal proveedor de productos hortofrutícolas de Estados Unidos, acumulando un $56 \%$ de la exportación total regional entre 2002 y 20I4. A continuación, tenemos a Chile (15\%), Costa Rica (7\%), Guatemala (6\%), Ecuador $(4 \%)$ y Perú $(4 \%)$. Los cuatro países restantes considerados, Honduras, Colombia, Brasil y Argentina, acumulan de manera conjunta poco más del 7\% del valor total de la exportación sectorial a Estados Unidos en el período bajo estudio. La escasa presencia de Argentina y Brasil en comparación con México, a pesar de que los tres son los principales exportadores de alimentos a nivel regional, entre otras cosas, se debe a las diferencias en sus patrones productivos. Si bien para México la venta de hortofrutícolas es protagónica en su canasta exportadora $(38,7 \%$ del valor total en 20I4), en el caso de Brasil y Argentina cumple un papel secundario frente a otros rubros clave como oleaginosas, carne, cereales y piensos.

Una vez obtenidos los registros relativos a rechazos de exportaciones de la base oAsIs para los países y años especificados (98.838 registros en total), se realizó una revisión detallada de cada uno de ellos, con el fin de seleccionar los correspondientes a productos en los capítulos 07 y 08 del SA. La necesidad de hacer dicha revisión es porque el tipo de mercancía afectada no es presentado en OASIS conforme a la nomenclatura del SA. Los registros seleccionados fueron sistematizados según año, país exportador, producto infractor y motivo (s) de rechazo. Respecto de lo último, OASIs identifica 262 violaciones posibles, las cuales se agruparon en: i) presencia de pesticidas, ii) productos sucios y/o descompuestos, iii) fallas en la manufactura, iv) falta de permiso adecuado de entrada, $v$ ) producto venenoso, vi) presencia de aditivos no seguros, y vii) incumplimiento de requisitos sobre etiquetado.

Entre los principales resultados obtenidos destaca, en primer lugar, que los envíos de productos hortofrutícolas latinoamericanos rechazados en la frontera de Estados Unidos en el período de 2002 a 2014 fueron en total II.576. México es el país de la región que ha enfrentado un más alto número de rechazos en el período estudiado con gran diferencia (Cuadro I). 


\section{CUADRO I}

Número de cargamentos rechazados de acuerdo a país y año, 2002-2014

\begin{tabular}{|c|c|c|c|c|c|c|c|c|c|c|c|}
\hline $\begin{array}{c}\text { AÑO/ } \\
\text { PAÍ́s }\end{array}$ & ARG. & BR. & CH. & COL. & C.R. & EC. & GUAT. & HOND. & MÉX. & PERÚ & TOTAL \\
\hline 2002 & 12 & 30 & 14 & 13 & 13 & 7 & 57 & 1 & 788 & 16 & 951 \\
\hline 2003 & 11 & 21 & 15 & 10 & 31 & 6 & 49 & 7 & 568 & 10 & 728 \\
\hline 2004 & 19 & 30 & 22 & 35 & 14 & 15 & 42 & 7 & 850 & 13 & 1.047 \\
\hline 2005 & 5 & 25 & 10 & 42 & 10 & 13 & 46 & 8 & 1.046 & 50 & 1.255 \\
\hline 2006 & 13 & 9 & 10 & 8 & 10 & 11 & 45 & 8 & 678 & 21 & 813 \\
\hline 2007 & 34 & 17 & 12 & 14 & 7 & 5 & 51 & 4 & 585 & 18 & 747 \\
\hline 2008 & 6 & 11 & 13 & 16 & 0 & 3 & 70 & 63 & 746 & 40 & 968 \\
\hline 2009 & 15 & 9 & 37 & 7 & 3 & 10 & 36 & 5 & 371 & 45 & 538 \\
\hline 2010 & 4 & 9 & 8 & 29 & 5 & 4 & 53 & 7 & 554 & 34 & 707 \\
\hline 2011 & 3 & 25 & 47 & 12 & 22 & 2 & 38 & 9 & 1.116 & 38 & 1.312 \\
\hline 2012 & 5 & 14 & 14 & 23 & 39 & 77 & 50 & 5 & 484 & 45 & 756 \\
\hline 2013 & 3 & 11 & 21 & 12 & 23 & 66 & 80 & 10 & 712 & 59 & 997 \\
\hline 2014 & 7 & 6 & 47 & 18 & 30 & 9 & 33 & 10 & 557 & 40 & 757 \\
\hline Total & 137 & 217 & 270 & 239 & 207 & 228 & 650 & 144 & 9.055 & 429 & 11.576 \\
\hline
\end{tabular}

Sin embargo, resulta muy difícil poder comparar el desempeño de los distintos países de la región, considerando solamente la cantidad total de rechazos. Esto porque aquellos que más exportan a Estados Unidos tienen, por supuesto, mayor probabilidad de un número más alto de rechazos (y viceversa). En consecuencia, calculamos un indicador $(\mathrm{T} i)$ que muestra la proporción entre la participación porcentual de cada país en el total de rechazos en el período bajo estudio y en el total de exportaciones. Un valor del indicador por debajo de uno significa que el país tiene una mayor participación en las exportaciones que en los rechazos, así como lo contrario si está por encima de uno (Cuadro 2). México es el país con el índice más alto $(\mathrm{I}, 37)$. En el extremo contrario están Chile $(0,16)$, Costa Rica $(0,25)$ y Ecuador $(0,46)$. 
CUADRO 2

Participación en exportaciones hortofrutícolas a Estados Unidos y en cargamentos rechazados por la FDA por país, 2002-2014*

\begin{tabular}{|c|c|c|c|c|c|}
\hline 至 & 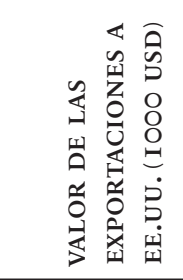 & 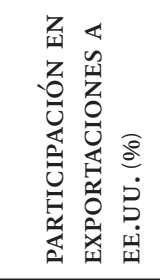 & 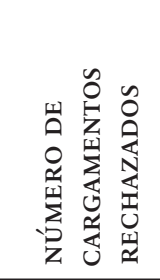 & 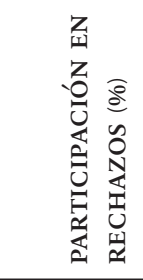 & $F$ \\
\hline Argentina & 1.598 .685 & 1,09 & 137 & 1,18 & 1,08 \\
\hline Brasil & 2.625 .524 & 1,79 & 217 & 1,87 & 1,04 \\
\hline Chile & 21.395 .166 & 14,63 & 270 & 2,33 & 0,16 \\
\hline Colombia & 3.109 .202 & 2,13 & 239 & 2,06 & 0,97 \\
\hline Costa Rica & 10.425 .163 & 7,13 & 207 & 1,79 & 0,25 \\
\hline Ecuador & 6.312 .290 & 4,32 & 228 & 1,97 & 0,46 \\
\hline Guatemala & 8.913 .478 & 6,09 & 650 & 5,62 & 0,92 \\
\hline Honduras & 3.123 .397 & 2,14 & 144 & 1,24 & 0,58 \\
\hline México & 83.409 .501 & 57,02 & 9.055 & 78,22 & 1,37 \\
\hline Perú & 5.365 .785 & 3,67 & 429 & 3,71 & 1,01 \\
\hline
\end{tabular}

"Porcentajes de participación consideran solamente los io países en el estudio.

El contenido específico de los cargamentos rechazados por país (según subpartidas del Sistema Armonizado) mostró coincidencia relativa con algunas de las partidas más comercializadas. En el Cuadro 3 se aprecia que los productos con una mayor participación dentro de las exportaciones hortofrutícolas desde América Latina a Estados Unidos, considerando sus denominaciones específicas conforme a las partidas del SA, son: vegetales, frescos o refrigerados (0709); bananas, incluyendo plátanos, frescos o secos
(0803); tomates, frescos o refrigerados (0702); dátiles, higos, piñas, aguacates, guayabas, mangos y mangostanes, frescos o secos (0804), y uvas, frescas o secas (0806). Dichas partidas acumularon más del $60 \%$ del valor total de las exportaciones en los capítulos 07 y 08 del SA de América Latina a Estados Unidos, entre 2002 y 20I4. Sin embargo, en los rechazos emergen otros productos con menor participación a nivel agregado, como las frutas y hortalizas procesadas, las leguminosas y los tubérculos (papas y camotes). En 
todo caso, aunque sería de gran interés, la mayoría de los países analizados, los resulta difícil establecer relaciones de rechazos están muy repartidos entre causalidad en este caso, dado que para distintos grupos de productos.

\section{CUADRO 3}

Productos más rechazados por país según códigos SA, 2002-2014*

\begin{tabular}{|c|l|l|l|l|l|}
\cline { 2 - 6 } \multicolumn{1}{c|}{} & ARGENTINA & BRASIL & CHILE & COLOMBIA & C. RICA \\
\hline 1 & $\begin{array}{l}\text { Uvas pasas } \\
(080620)\end{array}$ & $\begin{array}{l}\text { Pimientos } \\
(070960)\end{array}$ & $\begin{array}{l}\text { Uvas pasas } \\
(080620)\end{array}$ & $\begin{array}{l}\text { Especias } \\
(070990)\end{array}$ & $\begin{array}{l}\text { Especias } \\
(070990)\end{array}$ \\
\hline 2 & $\begin{array}{l}\text { Peras y } \\
\text { membrillos } \\
(080820)\end{array}$ & $\begin{array}{l}\text { Papayas } \\
(080720)\end{array}$ & $\begin{array}{l}\text { Duraznos } \\
(080930)\end{array}$ & $\begin{array}{l}\text { Frutos de } \\
\text { cáscara } \\
(080290)\end{array}$ & $\begin{array}{l}\text { Taro } \\
(071490)\end{array}$ \\
\hline 3 & $\begin{array}{l}\text { Frutas secas } \\
(081340)\end{array}$ & $\begin{array}{l}\text { Guayabas, } \\
\text { mangos y } \\
\text { mangostanes } \\
(080450)\end{array}$ & $\begin{array}{l}\text { Berries } \\
(081020)\end{array}$ & $\begin{array}{l}\text { Higo, } \\
\text { Guayaba en } \\
\text { mermelada } \\
(081190)\end{array}$ & $\begin{array}{l}\text { Patatas } \\
(070110)\end{array}$ \\
\hline 1 & $\begin{array}{l}\text { Guayabas, } \\
\text { mangos y } \\
\text { mangostanes } \\
(080450)\end{array}$ & $\begin{array}{l}\text { Arvejas } \\
(070810)\end{array}$ & $\begin{array}{l}\text { Melones } \\
(080719)\end{array}$ & $\begin{array}{l}\text { Hortalizas } \\
\text { deshidratadas } \\
(071290)\end{array}$ & $\begin{array}{l}\text { Pimientos } \\
(070960)\end{array}$ \\
\hline 2 & $\begin{array}{l}\text { Pepinos y } \\
\text { pepinillos } \\
(071140)\end{array}$ & $\begin{array}{l}\text { Frijoles } \\
(070820)\end{array}$ & $\begin{array}{l}\text { Quimbombó } \\
(070990)\end{array}$ & $\begin{array}{l}\text { Pimientos } \\
(070960)\end{array}$ & $\begin{array}{l}\text { Ají seco } \\
(071290)\end{array}$ \\
\hline 3 & $\begin{array}{l}\text { Taro (071490) } \\
\text { ECUADOR }\end{array}$ & $\begin{array}{l}\text { Especias } \\
(070990)\end{array}$ & $\begin{array}{l}\text { Hortalizas de } \\
\text { vaina }(070800)\end{array}$ & $\begin{array}{l}\text { Especias } \\
(070990)\end{array}$ & $\begin{array}{l}\text { Arvejas } \\
(070810)\end{array}$ \\
\hline
\end{tabular}

"Respecto a los códigos arancelarios, se consideró la versión 2002 del Sistema Armonizado.

Los motivos de incumplimiento asociados a los rechazos referidos se revisaron (Cuadro 4). De las violaciones registradas, el $4 \mathrm{I} \%$ está relacionado con aplicación inadecuada de pesticidas, $23 \%$ con que el producto esté descompuesto o sucio y $18 \%$ con que sea considerado como venenoso, vinculado esto último a la presencia de patógenos (Salmonella spp, Listeria spp, Shigella spp, entre otros). Estas violaciones son coincidentes con las recogidas 
por Buzby y Roberts (20II) para el período 1998-2004, como las principales cometidas por los países de ingreso medio en la exportación de alimentos a Estados Unidos. Asimismo, los resultados anteriores concuerdan con aquellos obtenidos por Artecona y Flores (2009). Estos autores demuestran que las razones de rechazo más frecuentes de alimentos por motivos técnicos en la frontera de Estados Unidos, específicamente en el caso de América Latina, tienen que ver con la inocuidad, lo que reduce las posibilidades de desvío de los cargamentos a terceros países (Baylis et al., 2010).

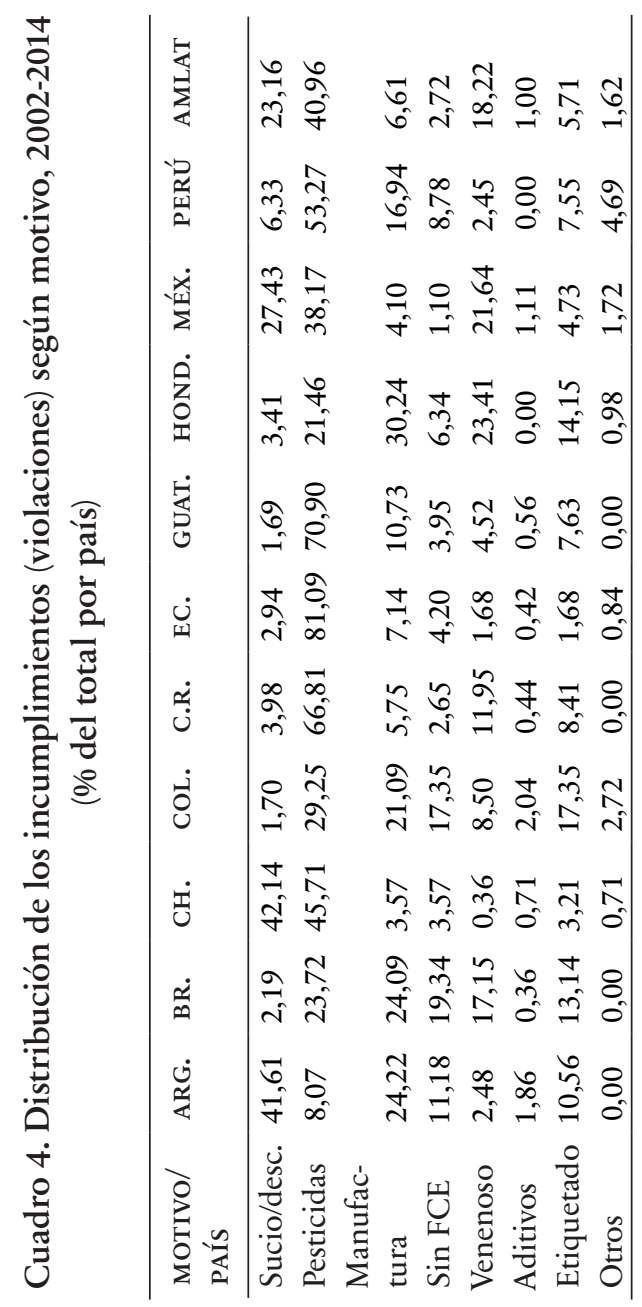


Si se analizan los incumplimientos a un nivel desagregado, vemos que hay algunas diferencias por país. Por ejemplo, para Argentina, Brasil y Colombia, las fallas en la fabricación y la falta de registro de FCE son la razón de alrededor del $40 \%$ de los rechazos. En México, un número importante está relacionado con productos venenosos. Mientras tanto, en Costa Rica, Ecuador y Guatemala, las violaciones por exceso de pesticidas son especialmente frecuentes. En Argentina y Chile, las negativas debido a la descomposición y la suciedad son importantes, y para ambos países hay hallazgos mínimos de productos venenosos. Estas diferencias tienen implicaciones claras para la política pública, ya que las estrategias deben enfocarse en aquellos eslabones de la cadena de producción, transporte y comercialización donde se detecta el incumplimiento.

En vista de los resultados obtenidos, en primer lugar podemos decir que existe heterogeneidad a nivel regional. Los dos casos más extremos son México y Chile. Si bien parte de la explicación del relativamente alto nivel de rechazos para México puede fundamentarse en su propio posicionamiento comercial, aparentemente existen otros factores que estarían influyendo al respecto. De hecho, México se sitúa como uno de los países a nivel mundial con mayor cantidad de rechazos de productos alimentarios en la frontera de Estados Unidos, según muestran estudios previos (Allen $e t$ al., 2008; Becker, 2010; Nguyen et al.,
2015). Los resultados obtenidos por Baylis et al. (2009) sugieren que esto podría estar en parte motivado por los intereses domésticos de Estados Unidos, en la medida que se quiera aminorar la competencia de productos similares procedentes de México. En efecto, México es el país geográficamente más próximo a Estados Unidos dentro de nuestra muestra, por lo que ambos tienen zonas productivas con climas similares en el mismo momento del año. Lo contrario sucede en otros casos como Chile, Perú, Argentina o Brasil, los que dada su ubicación en el Hemisferio Sur, tienen un ciclo productivo en contra estación respecto de Estados Unidos, por lo que la oferta es complementaria.

La presión del conocido como farm lobby es notoria en Estados Unidos, siendo muy activa, por ejemplo, en el contexto de las negociaciones comerciales. Conforme a Swinnen (2010), en las economías desarrolladas, el hecho de que la rentabilidad de otras actividades crezca más rápido que la del sector agrícola, genera incentivos para el intercambio de beneficios por apoyo entre productores y políticos, lo que conlleva altos retornos del lobbying. Por tanto, para los agricultores resulta económicamente más provechosa la generación de presión política para mantener un mercado protegido que realizar cambios internos en pro de mayor competitividad.

Por otro lado, la armonización puede ser una de las claves del bajo nivel de rechazos, al menos en casos 
como el de Chile. El acuerdo de libre comercio entre Chile y Estados Unidos, que ha estado en vigor desde 2004, incluye cooperación en materia de MSF. En este contexto, el USDA tiene agentes en los puertos chilenos que colaboran en realizar inspecciones in situ antes de embarque de productos junto con el Servicio Agrícola y Ganadero de Chile (Engler et al., 20I2).

No obstante, podríamos preguntarnos si un nivel más bajo de rechazos significa realmente un mayor cumplimiento de las medidas sanitarias y fitosanitarias establecidas por los Estados Unidos. Como se mencionó anteriormente, la FDA no puede inspeccionar en los puertos cada envío que llega a los Estados Unidos. En consecuencia, selecciona de antemano dónde se deben dirigir los controles. El nivel de riesgo que se asume para un envío es un criterio esencial. En este contexto, el historial de rechazos que registra el país de origen es relevante. Como consecuencia, el bajo nivel de rechazos puede verse influido por la menor cantidad de inspecciones in situ, dado lo que podríamos llamar un "efecto de reputación”. No obstante, con lo anterior no se pretenden ignorar los esfuerzos de cada país por mejorar su sistema de control de alimentos.

Respecto de esto último, aunque no se evidencia una relación definitiva entre capacidades del sistema de control de alimentos y rechazos, algunos casos particulares son destacables. Por ejemplo, Chile y Costa Rica tienen un positivo desempeño en ambos aspectos, comparados con otros países de la región. En particular, Chile tiene un sistema de control de alimentos integrado, con una red de instituciones especializadas, cuyas capacidades son reconocidas. Por otra parte, el país también ha sido muy activo en el número de medidas técnicas notificadas a la омс, lo que sugiere la presencia de las capacidades necesarias para poder diseñar, implementar e inspeccionar. No obstante, para otros países como México o Perú -también destacadamente activos en número de notificacionesno podemos realizar el mismo análisis.

\section{Comentarios FinAles}

En América Latina, donde muchos países son exportadores netos de alimentos, el fortalecimiento de los sistemas de control es de vital importancia, no tan solo para evitar que algunos productos puedan ocasionar daños a la salud pública, animal y vegetal, sino como un medio para fomentar el comercio internacional. Considerando lo anterior, los gobiernos de la región deben visualizar la inocuidad alimentaria como un tema trascendental, comprometiendo mayores recursos en primera instancia para acercar la realidad de cada uno de estos países a los estándares esperados de países 
desarrollados, mejorando los sistemas de control de alimentos y, más aún, la legislación y los procesos de inspección desde una perspectiva de análisis de riesgo, para reducir gastos en salud pública derivados de Enfermedades Transmitidas por Alimentos y recursos destinados a mitigaciones por presencia de nuevas plagas y contaminantes; así como la imposibilidad de ingresar los cargamentos rechazados en los mercados internacionales de destino.

Este es el marco a partir del cual se deberían ampliar y mejorar las capacidades humanas, institucionales y de infraestructura necesarias para responder con más eficacia y rapidez a los nuevos riesgos e innovaciones del sector alimentario; consolidándose la confianza de los consumidores, tanto de los mercados nacionales como internacionales de toda la región.

Los resultados obtenidos en el presente artículo muestran que el número de rechazos de cargamentos de productos hortofrutícolas en las fronteras de Estados Unidos presenta importantes diferencias entre países latinoamericanos, no justificadas de manera íntegra por el valor relativo de sus exportaciones. En este sentido, los de México, Chile y Costa Rica son los casos más llamativos. El primero de ellos por su alto número de rechazos y los otros dos por tener una participación relativamente baja en los mismos.

Por otra parte, los vegetales, las uvas, las frutas y hortalizas procesadas, las leguminosas y algunos tubérculos, como papas y camotes, destacan en lo que respecta al contenido de los cargamentos más rechazados. La aplicación inadecuada de pesticidas, que el producto esté descompuesto o sucio, y que sea considerado como venenoso por presencia de patógenos, son los tipos de violaciones detectadas con mayor frecuencia a nivel agregado. Sin embargo, se observó que lo anterior no es homogéneo para todos los países considerados en el estudio.

Respecto de las causas detrás de los resultados obtenidos, que justificarían los distintos niveles de rechazo, no existe una sola interpretación posible. Hay factores que parecen interactuar, lo que hace que se ajusten a algunos casos, pero no a otros. Entre estos podemos señalar: la intensidad del comercio, la capacidad del sistema nacional de control de alimentos, el desempeño regulatorio en medidas técnicas, el nivel de armonización con los requisitos impuestos por Estados Unidos, la "reputación” del país basada en su historial de incumplimientos y rechazos, así como la complementariedad/competencia con la oferta interna estadounidense.

Finalmente, señalar que una de las limitaciones del presente estudio es que no fue posible, con la información disponible, determinar la cantidad exacta de alimentos rechazados y, por ende, las pérdidas económicas relativas, pues solo se contó con notificaciones de rechazo correspondientes a cada incumplimiento, sin especificar otras unidades. Adicionalmente, se desconocen los formatos de venta de 
los productos rechazados, dato importante al buscar valorizar, pues algunos alcanzan precios significativamente superiores a otros. Aun así, estos datos podrían ser estimados bajo cálculos indirectos basados en suposiciones de capacidad de cargamentos rechazados, calidad de los productos y valores de mercado de las mercancías notificadas, lo que ha sido realizado hasta ahora de forma pionera para el caso específico de Chile (Boza et al., 20I8).

\section{Bibliografía}

Allen, A.J.; Myles, A.E.; Shaik, S., y Yeboah, O. (2008). "An analysis of trends in food imports refusals in the United States". Journal of Food Distribution Research, vol. 39, pp. 5-IO.

Artecona, R. y Flores, F. (2009). Observatorio del control aduanero a las importaciones de Estados Unidos: estándares técnicos. Washington DC: CEPAL, p. 25.

Baylis, K.; Martens, A., y Nogueira, L. (2009). "What drives food import refusals?”. American Journal of Agricultural Economics, vol. 9I, pp. I477-I483.

Baylis, K.; Nogueira, L., y Pace, K. (2010). "Food import refusals: evidence from the European Union". American Journal of Agricultural Economics, vol. 93, pp. 566-572.

Becker, G.S. (2010). US food and agricultural imports: safeguards and selected issues. Washington DC: Congressional Research Services, p. I7.

Beghin, J. y Melatos, M. (20I2). "The trade and welfare impacts of Australian quarantine policies: the case of pigmeat". World Economy, vol. 35, pp. I006-IO2I.

Boza, S. y Fernández, F. (2016). "World Trade Organization members' participation in mechanisms under the Sanitary and Phytosanitary Agreement”.
International Journal of Global Trade and Markets, vol. 9, No. 3, pp. 2I2-227.

Boza, S. y Muñoz, J. (20I7). "Factors underlying sanitary and phytosanitary regulation for food and agricultural imports notified by wTо members". The Journal of International Trade \& Economic Development, vol. 26, No. 6, pp. 712-723.

Boza, S.; Rivers, R., y Rozas, J. (20I4). "Participación y capacidades de ALC en medidas sanitarias y fitosanitarias". Revista Puentes, vol. I5, No. I, pp. I4-I7.

Boza, S.; Rivers, R.; Rozas, J., y Muñoz, J. (20I8). "Implications of non-compliance with Technical Non-Tariff Measures: The case of Chilean food related export refusals at the United States border", en De Melo, J. y Nicita, A. (eds.), Non-Tariff Measures: Economic Assessment and Policy Options for Development. Ginebra: United Nations Conference on Trade and Development, pp. 225-253.

Brooks, N.; Buzby, J.C., y Regmi, A. (2009). "Globalization and evolving preferences drive U.S. food-import growth". Journal of Food Distribution Research, vol. 40, pp.39-46. 
Buzby, J.C. y Regmi, A. (2009). "FDA refusals of food imports by exporting country group". Choices, vol. 24, pp. II-I5

Buzby,J.C.y Roberts, D. (20II). "Food trade and food safety violations: what can we learn from import refusal data?". American Journal of Agricultural Economics, vol. 93, pp. 560-565.

Buzby, J.C.; Unnevehr, L.J., y Roberts, D. (2008). Food safety and imports: an analysis of FDA food-related import refusal reports. Washington DC: U.S Department of Agriculture Economic Research Service. p. 4I.

CEPAL, FAO, IICA (20I5). Perspectivas de la Agricultura y del Desarrollo Rural en las Américas: una Mirada hacia América Latina y el Caribe. San José de C. R.: IICA, p. 2 I2.

Crivelli, P. y Gröschl, J. (20I2). "SPS measures and trade: implementation matters". World Trade Organization Staff Working Paper ERSD-20I 2-05.

Disdier, A-C. y Fontagné, L. (2010). “Trade impact of European measures on GMOs condemned by WTO panel". Review of World Economics, vol. I46, pp. 495-5I4.

Disdier, A-C. y Marette, S. (2010). "The combination of gravity and welfare approaches for evaluating non-tariff measures". American Journal of Agricultural Economics, vol. 92, pp. 713-726.

Engler, A.; Nahuelhual, L.; Cofré, G., y Barrena, J. (20I2). "How far from harmonization are sanitary, phytosanitary and quality-related standards? An exporter's perception approach". Food Policy, vol. 37, pp. I62-170.
Ferro, E.; Otsuki, T., y Wilson, J.S. (2015). "The effect of product standards on agricultural exports". Food Policy, vol. 50, pp. 68-79.

Food and Agriculture Organization of the United Nations (FAO) (2007). Fortalecimiento de los sistemas nacionales de control de los alimentos. Directrices para evaluar las necesidades de fortalecimiento de la capacidad. Roma: FAO, p. 165 .

Food and Agriculture Organization of the United Nations (FAO) (20IO). Documento de Trabajo del Foro Regional "Políticas de Seguridad Alimentaria e Inocuidad y Calidad Alimentaria: Casos Exitosos de Integración”.FAO/RLC: Santiago de Chile.

Food and Agriculture Organization of the United Nations (FAO) (20I2). Proyecto FAO TCP/RLA/3213: Asistencia para el diseño y/o fortalecimiento de políticas de inocuidad de alimentos para los países de la región. FAO/RLC: Santiago de Chile.

Grant, J. y Anders, S. (20II). "Trade deflection arising from U.S. import refusals and detentions in fishery and seafood trade". American Journal of Agricultural Economics, vol. 92, pp. 713-726.

Grundke, R., y Moser, Ch. (20I4). "Hidden protectionism? Evidence from non-tariff barriers to trade in the United States", KOF Working Papers.

Hobbs, J.E. (20I0), "Public and private standards for food safety and quality: international trade implications". Estey Centre Journal International Law \& Trade Policy, vol. II, pp. I36-I52. 
Hoekman, B. y Nicita, A. (2008). “Trade policy, trade costs, and developing country trade". World Bank Policy Research Working Paper, 4797.

Jouanjean, M-A (2012). "Standards, reputation, and trade: evidence from US horticultural import refusals". World Trade Review, vol. II, pp. 438-46I.

Nguyen, L.; Nelson, R.G., y Wilson, N.L. (2015). Triggering factors for US import refusals. Southern Agricultural Economics Association 2015 Annual Meeting, January $3 \mathrm{I}^{\text {st }}$-February $3^{\text {rd }}$. Atlanta, Georgia, US.

Nicita, A. y Gourdon, J. (2013). “A preliminary analysis on newly collected data on non-tariff measures". UNCTAD Policy Issues in International Trade and Commodities Study Series, No. 53.

OECD, FAO (2015). OECD-FAO Agricultural Outlook 20I5. Paris: OECD, p. I45.

Penello, D. (20I4). "Study of average effects of non-tariff measures on trade imports". UNCTAD Policy Issues in International Trade and Commodities Research Study Series, No. 66.

Santana, R., Jackson, L.A. (20I2). "Identifying non-tariff barriers: evolution of multilateral instruments and evidence from the disputes (1948-20II)". World Trade Review, vol. II, pp. 462-478.

Scallan, E., Hoekstra, R.M.; Angulo, F.J.; Tauxe, R.V.; Widdowson, M-A; Roy, S.L.; Jones, J.L., y Griffin, P.M. (20II). "Foodborne illness acquired in the United States: Major pathogens”.Emerging Infectious Diseases Journal, vol. I7, No. I, pp. 7-I5.

Swinnen, J. (2010)."The Political Economy of Agricultural and Food Policies: Recent Contributions, New Insights, and Areas for Further Research". Applied Economic Perspectives and Policy, vol. 32, Issue I, pp. 33-58.

UNCTAD (2013). "Non-tariff measures to trade: economic and policy issues for developing countries”. Geneva: United Nations Publications, p. 108.

Van Tongeren, F.; Beghin, J., y Marette, S. (2009). "A cost-benefit framework for the assessment of non-tariff measures in agro-food trade”. OECD Food Agriculture and Fisheries Working Papers, No. 2I.

Wilson, N., y Bray, V. (20I0). It happened all at once: switching regressions, gravity models and food safety. AAEA, CAES, WAEA Joint Annual Meeting, July $25^{\text {th }}$ July $27^{\text {th }}$, Denver, Colorado, US. 